\title{
TIBIAL DIAPHYSEAL FRACTURES IN CHILDREN
}

\author{
Cláudio Santill , Caetano Maria de Oliveira Gomes, Miguel Akkari , Gilberto Waisberg, \\ Susana dos Reis Braga, Wilson Lino Junior, Fabrício Guimarães Santos
}

\section{ABSTRACT}

Tibial diaphyseal fractures in children and adolescents are relatively common injuries and often evolve with good results when treated through traditional methods of conservative treatment. Their elevated frequency is due to the high degree of exposure of children in physical activities and also to the topographic location, exposing them to direct or indirect trauma. The approach used should consider and respect some features regarding age, place of affection (whether proximal or distal), type of fracture and therapy. The anatomical advantage of a thicker periosteum and flexibility when submitted to angular impacts can provide younger children with greater stability and, consequently, increases their chances of a better prognosis than older children and adolescents. In the latter, the degree of exposure to highenergy trauma and the greater complexity and severity of injuries have caused the recent trend towards stabilization surgery to become more common. Frequent complications in the evolution of fractures in adults such as infection, delayed union and nonunion are much less common in children, although the risk of occurrence of compartment syndrome is an event that requires attention, especially with plaster.

Keywords: Tibial fractures. Child. Leg injuries. Orthopedics.

Citation: Santili C, Gomes CMO, Akkari M, Waisberg G, Braga SR, Lino Junior W, Santos FG. Tibial diaphyseal fractures in children. Acta Ortop Bras. [online]. 2010; 18(1):44-8. Available from URL: http://www.scielo.br/aob.

\section{GENERAL CONSIDERATIONS}

Tibia fractures in children and adolescents constitute the most common lower-extremity fractures, representing 10 to $15 \%$ of all fractures in the pediatric population. In relation to adults, they have the significant particularity of consolidating quickly and favorably with closed treatment, and also present few complications such as delay in consolidation and pseudarthrosis. ${ }^{1-6}$ Fractures in this region are much more common in children that are still learning to walk. The fractures usually result from lowenergy trauma, causing fractures with smaller deviations than in adolescents, as bone resistance to direct impact trauma and to torsional trauma increases with age.

Fractures caused by high-energy trauma are more common in adolescence, a period in which fractures with tibia and ipsilateral fibular displacement are associated with soft tissue injury and make the treatment more complex, besides influencing the prognosis. ${ }^{1-6}$

\section{EPIDEMIOLOGY}

Tibia fractures are the third most common type and represent from 10 to $15 \%$ of pediatric fractures. The peak of affection occurs on an average at the age of eight, ${ }^{2-8}$ whereas the ratio of incidence between boys and girls is $2: 1 .^{1}$
Approximately 6 to $10 \%$ of tibia fractures are exposed, and $70 \%$ of them occur in an isolated manner, while $30 \%$ are associated with ipsilateral fibular fracture. , $^{1,8}$

Twenty-six out of every one hundred (26\%) children who are victims of abuse resulting in fractures present tibia fractures, making this segment the second most frequently fractured bone in beaten children. ${ }^{1,6}$

Up to the age of seven years, the most common traumas reported originate from domestic and school accidents. From five to ten years, cases of children run over by vehicles are important causes of fractures, while from seven to fourteen years of age, accidents caused from falling off bicycles, sports (soccer, volleyball, basketball, etc.), skateboarding and the so-called radical sports are the most frequent causal factors of tibia fractures in this age bracket. Epiphyseal fractures and detachments occur as a consequence of forces applied indirectly at the site of the affected lower limb. Direct traumas produce transverse, simple oblique or comminuted fractures. The torsion mechanism with angulation usually results in a long oblique or spiral fracture..$^{4,6,7}$

\section{ANATOMY}

The anteromedial region of the tibia is subcutaneous and without underlying musculature for its protection. ${ }^{1,6}$

All the authors declare that there is no potential conflict of interest referring to this article.

Department of Orthopedics and Traumatology of the Faculdade de Ciências Médicas da Santa Casa de São Paulo

Study conducted in the Pediatric Orthopedics Group of the Department of Orthopedics and Traumatology of the Central Hospital of Faculdade de Ciências Médicas da Santa Casa de São Paulo, "Pavilhão Fernandinho Simonsen". Director: Prof. Dr. Osmar Avanzi

Mailing address: Departamento de Ortopedia e Traumatologia da Santa Casa de Misericórdia de São Paulo - Grupo de Ortopedia e Traumatologia Pediátrica do Hospital e da Faculdade de Ciências Médicas da Santa Casa de São Paulo - Rua Cesário Motta Junior 112 - CEP: 01277-900. Vila Buarque - São Paulo-SP. Brazil.

Site: www.ortopediatrica.com.br. E-mail: ortopediatrica@yahoo.com.br

Article received on $1 / 30 / 09$ and approved on 6/29/09 
There are three ossification centers that form the tibia:

1 - Proximal epiphysis - The proximal secondary ossification center appears soon after birth with later closure at 16 years of age.

2 - Diaphyseal - It ossifies in the seventh week of gestation and expands proximally and distally.

3 - Distal epiphysis - The distal secondary ossification center appears in the second year with later closure at 15 years of age.

The malleolus and medial tubercle can appear as separate ossification centers and should not be confused with a fracture. ${ }^{1}$

The nutrient artery originates from the posterior tibial artery and penetrates in the posterolateral cortical bone, distally. In the intramedullary canal, it gives rise to three ascending and one descending branch, which in turn originate endosteal vascularization and anastomose with the periosteal vessels that protrude from the anterior tibial artery. This artery in turn is particularly vulnerable to lesions as it passes through the hiatus of the interosseous membrane. ${ }^{1}$

The common fibular describes its course around the fibular neck that is almost subcutaneous in this region and is particularly vulnerable to injuries by direct trauma or by indirect traction mechanism.

\section{Fascial compartments}

The leg has four fascial compartments. ${ }^{6}$

1 - Anterior - Contains the extensor digitorum longus and extensor hallucis longus muscles and the tibialis anterior muscle; anterior tibial artery and the deep fibular nerve.

2 - Lateral - Contains the fibularis longus and fibularis brevis muscles and the superficial fibular nerve.

3 - Superficial posterior - Contains the soleus and gastrocnemius muscles.

4 - Deep posterior - Contains the flexor digitorum longus and the flexor hallucis longus, tibialis posterior, posterior tibial artery, fibular artery and tibial nerve.

\section{LESION MECHANISM}

Isolated fractures of the tibia generally result from direct trauma. ${ }^{1}$ Of all the tibial fractures with intact fibula, $81 \%$ are caused by indirect rotational forces.

Fifty percent of tibial and ipsilateral fibular fractures results from trauma caused by individuals being run over by a motor vehicle. ${ }^{1}$

\section{CLASSIFICATION}

A. Proximal shaft fracture of the tibia and fibula

B. Proximal metaphyseal fracture of the tibia

C. Diaphyseal fracture of the tibia and fibula

1. diaphyseal fracture of the tibia

2. diaphyseal fracture of the tibia and fibula

3. diaphyseal fracture of the fibula

D. Distal metaphyseal fracture of tibia

E. Distal shaft fracture of the tibia and fibula

\section{RADIOGRAPHIC EVALUATION}

The anterior-posterior ${ }^{1}$ (AP) and lateral views of the leg and knee should be obtained. The AP, lateral and true AP views of the ankle should be performed to rule out concomitant lesion of the ankle. Comparative $x$-rays of the unimpaired contralateral limb might be necessary in rare cases.

Bone scintillography with technetium or magnetic resonance might be requested in some cases to rule out a concealed fracture in the appropriate clinical context.

\section{TYPES OF FRACTURE AND TREATMENT}

"Most pediatric fractures of the tibia and of the fibula are not Acta Ortop Bras. 2010; 18(1):44-8 complicated and can be treated by simple manipulation and immobilization, especially in the absence of deviation or if it is minimal." Koval and Zuckerman. ${ }^{1}$

\section{Proximal metaphyseal}

Proximal metaphyseal greenstick fractures with acceptable deviation or without deviation, are treated in a closed manner by means of immobilization with a plaster cruropodalic cast and varus modeling. Depending on the patient's age, such fractures are kept immobilized by a period of 21 to 35 days, keeping the knee in flexion of $10-15^{\circ}$.

Zionts and MacEwen ${ }^{9}$ monitored seven children with deformity in progressive valgus of the tibia after metaphyseal fracture and concluded that the best attitude would be, "to wait and see". The highest percentage of the deformity occurred during the first year. The tibia continued to deform at a slower pace up to 17 months after the injury. Totally spontaneous clinical correction occurred in six patients. After the average follow-up period of 39 months; all the children radiographically presented less than 10 degrees of residual deformity.

In angulated or deviated greenstick fractures, the performance of closed reduction is recommended whenever possible with the help of an image intensifier, under general anesthesia, followed by immobilization with plaster cruropodalic cast with the knee in total extension and a movement in varus applied on the knee to avoid valgus collapse. The immobilization is maintained for four to six weeks, depending on the age of the child. It is inadvisable to use local anesthesia or simple sedation in order to achieve reduction and to make the plaster cast in a safe and trouble free manner. ${ }^{1,5-8}$

Rare metaphyseal fractures become irreducible or unstable when treated by closed methods and this may occur due to the interposition of soft, ligamentary or tendinous (knee flexors) parts. In these cases the recommended treatment is surgical reduction and fixation by means of Kirschner wires, associated with plaster cast immobilization, for a period of 45-60 days. As these fractures are located in a strongly vascularized area, comprised of spongy bone, they consolidate very quickly.

In the post-plaster cast period, physiotherapy should be indicated for muscular strengthening of the affected limb, with particular attention to the quadriceps, as well as to gain and maintain the range of movement of the knee and ankle.

Robert et al. ${ }^{10}$, in an analysis of 25 children, reported 12 children with greenstick or complete fracture and 11 with progressive valgus. There were no complications in the tubercle fractures, and in three fractures, there was compensation with distal growth of the shaft. Four children were submitted to corrective osteotomy, there were two recurrences of the deformity and two cases of compartment syndrome.

Several authors affirm that the treatment of proximal metaphyseal fractures consolidated in valgus consists of observation and clinical follow-up due to the common occurrence of the spontaneous disappearance of the problem with the development of an "S" shaped tibia. ${ }^{6,11,12}$ Persistent deformity should only be corrected surgically in puberty. 4,10

\section{Diaphyseal fractures}

Fractures of the diaphysis of the tibia or fibula are responsible for approximately 4 to $5 \%$ of all pediatric fractures ${ }^{(2,4)}$. In general, these fractures fall into three categories:

- Without deviation

- Oblique or spiral

- Transverse and comminuted with deviation 
Less than $4 \%$ of these fractures require surgical treatment, and in $70 \%$ of the cases they are isolated tibial fractures, which maintains leg length to a certain extent manner, yet predisposes to angular deformity in valgus. ${ }^{5}$

The tibia has limited remodeling potential. Rotational deformities do not undergo remodeling. Nevertheless, in clinical practice it is observed that the deformity in external rotation is tolerated better that that in internal rotation. ${ }^{13}$

During growth, babies and infants can correct approximately $50 \%$ of post-fracture residual angulation. In children over 10 years of age, there will only be an improvement of the poor axial alignment in $25 \%$ of the cases. Hansen et al. ${ }^{14}$ communicated that just $13.5 \%$ of their patients achieved correction of angular deformity with subsequent growth, while Shannak ${ }^{13}$ observed that $1 / 3$ of the children, with more than 10 degrees of angulation upon consolidation, persisted with some degree of defective consolidation until the final evaluation of the follow-up, which was from three to ten years. And he concluded that, in general, defective alignment in varus appears to remodel more efficiently than deformity in valgus.

Ipsilateral tibial and fibular fractures exhibit considerable instability, and reductions with less than $50 \%$ of contact among tibia fragments and angulations above five degrees of valgus and varus should not be accepted. ${ }^{5-8,15}$

Isolated tibial diaphyseal fractures tend to deviate in varus, while those that involve the tibia and fibula tend to deviate in valgus, with the risk of shortening and recurvatum. ${ }^{1}$

In fractures without deviation or with minimum deviation within acceptable limits (valgus $5^{\circ}$, varus 5-10', antecurvatum 5-10 ${ }^{\circ}$, recurvatum $0-5^{\circ}$, shortening $20-25 \mathrm{~mm}$ and rotation $5^{\circ}$ ), the treatment indicated is immediate plaster cruropodalic cast immobilization with the knee in flexion from 10 to $15^{\circ} .{ }^{5-8}$

In cases in which fractures exhibit important edema, the recommended treatment is immobilization with plaster cruropodalic splint for seven to 10 days and subsequent conversion to a plaster cast, with light contact with ground allowed with the aid of two crutches when possible, after four weeks. Between the fourth and sixth weeks, the plaster cruropodalic cast can be converted into a plaster boot for gait in transverse and short oblique fractures and those located between the medial third and distal bones. Radiographic control is performed in the first and third weeks and physiotherapy should be recommended in children from pre-adolescence. Yang and Letts ${ }^{16}$ observed 95 children including 61 boys and 34 girls. The distal third of the leg was affected in 69 children, the medial third in 17 and proximal fractures in nine children. In relation to lines, 81 fractures were spiral or oblique, 11 transverse and three comminuted. After the follow-up time of 38.8 months they verified that the consolidation time was five weeks (ranging from 2 to 16 weeks). Of the 95 fractures, 19 were immobilized with plaster cruropodalic cast and 70 presented dislocated fractures with angular deformity having been submitted to closed reduction and immobilization with PTB (patellar tendon-bearing) type plaster. The six fractures resulting from traffic accidents had serious lesions and were submitted to open reduction followed by internal or external fixation. Indirect trauma was the most common mechanism, with 39 fractures resulting from skiing/skating accidents, 38 from falls, three from child abuse and 15 from other road accidents.

Unstable, extensive comminuted, multiple or contralateral leg fractures, polytraumatized fractures (cephalorachidian, thoracic and/ or abdominal trauma) and open fractures, benefit from surgical treatment with temporary or permanent stabilization, to facilitate the handling of the child.
The type of osteosynthesis to be used in children is still highly controversial today and not yet totally accepted consensually, with a great deal of respect shown for the experience and common sense of each surgeon. Plates produce good immobilization, yet require incisions that are somewhat extensive and additional procedures to remove them. Their use is not recommended in patients under 12 years of age due to the excessive growth potential that they can produce, due to the stimulus to the fracture union plates. ${ }^{17}$

As regards the use of rigid intramedullary nails after closed reduction, it is known that although they constitute a very safe method from the surgical point of view, they should only be used in adolescents, after the shaft closing age or very close to it. Nonetheless, in adolescence they can already be used with the same criteria as for adults. Srivastava et al. ${ }^{18}$ studied 24 high-energy trauma fractures in 24 children. The mean age was 11 years, ranging from 4.6 to 16.4 years. They analyzed eight closed fractures in which the mean consolidation time was 21.5 weeks and 16 exposed fractures with 20.3 weeks, whereas the general mean consolidation time was 20.4 weeks. The indications for surgical treatment with stabilization with intramedullary nails were unstable and exposed fractures, polytrauma, floating knee, CET, failure in closed reduction and compartment syndrome. As advantages of the method they obtained: immediate stabilization, early mobility, reduced additional aggression to the soft parts, low risk of infection and refracture, besides reduction in the time of return to activities. As regards complications, they reported two compartment syndromes, two deep infections and two defective consolidations. Now Lascombes et al. ${ }^{19}$ concernedly analyzed the tendencies of use and overuse of nails in children and as advantages referred to early mobilization in the immediate postoperative period. It is a minimally invasive surgery and there is no growth plate aggression. As complications they refer to errors of technique, failure in consolidation, pseudarthrosis, osteomyelitis, refracture and limb length discrepancy. They report one percent of non-consolidation in comminuting fractures and discrepancies of up to one centimeter in four patients in 18 floating knees.

With the advances of the metallurgical industry and consequent refinement and improvement of materials such as flexible titanium nails, it is now possible to perform closed reduction, with the support of an image intensifier and subsequent closed intramedullary nailing, thus reducing surgical aggression to the fracture focus and the risks of lifting off the periosteum, besides diminishing the risk of postoperative infection.

Vallamshetta et al. ${ }^{20}$ studied 54 children, 43 male and 11 female, with 56 unstable fractures treated with flexible intramedullary nails. The mean age was 12 years (ranging from 4 to 16 years); 35 fractures had been caused by high-energy trauma, and 13 were exposed. Most of the fractures (68\%) were located in the medial third. The consolidation time was ten weeks (ranging from 7 to 18 weeks) and the follow-up period 11 months (ranging from 8 to 17 months). As complications they observed residual angulation of the tibia, discrepancy of limb length, deep infection and fixation failures, and that in spite of this, the children all showed excellent functional result. Kubiak et al. ${ }^{21}$ conducted a retrospective review, evaluating the differences in the development of results of tibial fractures in children treated with external fixation versus flexible nails. They reviewed 31 patients that needed surgical treatment of the fractures, treating 16 tibias with flexible nails and 15 with external fixation. They evaluated the rates of complications and the need for further surgery clinically and radiographically. There was no statistical difference 
between the two groups. The main conclusions of the study included a significantly lower number in relation to the consolidation time for patients in the flexible nail group (7 versus 18 weeks), and a significant rate of bone complications related to the group treated with external fixator. Without any randomized prospective studies comparing flexible nails and external fixation, there is no established consensus on the best fixation of pediatric tibial fractures. However, there appears to be a tendency toward the treatment of these fractures with flexible nails whenever possible.

El-adi et al. ${ }^{22}$ prospectively evaluated 48 femoral fractures and 25 tibial fractures, treated with flexible intramedullary nails. In spite of complications like vicious consolidation, discrepancy of the lower limbs and irritation in the soft parts related to the entrance points of the nails, all the cases obtained good or satisfactory results.

"Flexible intramedullary fixation is an easy and effective method in the treatment of unstable exposed and/or closed tibial fractures in children". ${ }^{20}$

\section{Open fractures}

Open fractures of the tibia in children are a result of high-energy trauma, and are associated with other injuries in 25 to $50 \%$ of the cases. 5,6,23,24

The initial treatment consists of the administration of intravenous antibiotics and tetanus prophylaxis; this is followed by copious irrigation and thorough cleansing of the wound. 4,5,23-26 After adequate debridement, first-degree open and clean fractures can have the wound loosely sutured, and a drainage tube can be fixed, but in most cases, the wounds should be left open, as among other reasons, they might be submitted to further debridement. ${ }^{25}$

Kreder and Armstrong ${ }^{24}$ noticed that a delay of more than six hours in surgical cleaning was correlated to $25 \%$ of infection in comparison with $12 \%$ for patients operated within a time limit of up to six hours. Other authors, Song et al. ${ }^{26}$ and Blazier and Barnes, ${ }^{27}$ observed that age is the most significant prognostic indicator. They propose that children under 12 years of age need less aggressive surgical treatment, as their injuries heal faster, they have lower percentages of infection and present fewer complications than children above this age. ${ }^{26,27}$

In open fractures the use of external fixators is a relatively simple procedure with the advantage of maintaining the reduction and stability of the fracture, besides favoring easy access for the cleaning of wounds. Some models allow patients to walk at an early stage and do not cause limitation of joint movement amplitude, both of the knee and of the ankle. They present low morbidity provided maximum care is taken with possible infections along the course of the pins, to avoid deep infection. Bartlett et al. ${ }^{28}$ studied 23 exposed fractures in children aged between 3.5 and 14.5 years, with 18 boys and 5 girls. The types of fracture were: 6 second-degree fractures, 8 type IIIA fractures and 9 type IIIB fractures with 7 comminuting butterfly-wing fractures. The lesion mechanisms were: all the fractures caused by running over by motor vehicles except for two, one of which was the fall of a motor vehicle and the other a collision between a motorcycle and motor vehicle. Five children had other associated lesions (CET, fracture of the acetabulum, femur, humerus, mandible, maxilla, and severe disorders of knee ligaments).

The Orthofix monolateral and AO fixators or even Wagner bone lengtheners are those of preferential use due to the ease of installation, and versatility when the correction of any secondary deviations is necessary. Above all, they make it possible to reduce and maintain the reduction as they are extremely resistant to longitudinal traction forces, compression and angulation.

Circular fixators and rigid models are not intended for routine use and any indication of these devices in the treatment of traumatic lesions in children is exceptional and should be practiced with prudence. Buckley et al ${ }^{29}$ studied 20 children with severe exposed fractures of Type III (7 IIIA, 10 IIIB, 3 IIIC), of which 11 were comminuted fractures, three segment, one transverse and one spiral. Seventeen were in the diaphyseal region, two were proximal metaphyseal and one a distal metaphyseal fracture. The age range of the children was from 2 years and 11 months to 16 years and 2 months (mean age 9 years) with the involvement of 16 boys and 4 girls. The most common lesion mechanism was car accident in 12 cases. The treatment employed was the external fixator in 15 fractures, plaster cast in three, an external fixator associated with minimal internal fixation (interfragmentary screw) and one open reduction and fixation with plate. The complications encountered were: three vascular lesions (two required a graft), two nerve lesions and three cases of osteomyelitis.

In a recent review, Gougolias et al. ${ }^{30}$ suggest that flexible intramedullary nails are an alternative, yet further studies are necessary to determine their best indication in these cases.

\section{Distal metaphyseal fractures}

Treatment is conservative when no unacceptable deviation is observed. Isolated fractures of the tibia or associated with distal fracture of the fibula, can sometimes present deviation and are generally in recurvatum or with lateral angulations.

The vast majority of fractures with deviation require the performance of closed reduction under general anesthesia followed by immobilization for 30 to 45 days, depending on the age of the child, the plaster cast can be of the boot type or longer, reaching above the knee in the case of instability and consequent risk of loss of the reduction. Immobilization is mandatory if the recurvatum is accentuated and with a high possibility of secondary deviation inside the actual cast, including the foot in plantar flexion for a period of three weeks and after this in the anatomical position of $90^{\circ}$ in relation to the leg axis. ${ }^{1,5,6}$ In unstable fractures or fractures with suspicion of interposition where reduction is impossible, the recommended treatment is open exploration; removal of the interposed tissue and after reduction fixation with smooth Kirschner wires with tapered edge or compression screws avoiding crossing the growth plate.

\section{IMMEDIATE COMPLICATIONS}

\section{Compartment syndrome}

Compartment syndrome may sometimes occur in fractures of the proximal third, particularly in the presence of severe trauma affecting the soft parts, after prolonged traumatic compressions or those caused by very tight plaster casts, especially when they are donned in the presence of intense edema or when the child fails to keep the limb in an elevated position. Symptoms and clinical signs, when present, can be confirmed by measuring the compartment pressure and imply urgent measures, which can range from simple removal of the cast and elevation of the limb to decompressive fasciotomy and use of an external fixator to guarantee bone stabilization and to provide freedom to control and care for the soft parts.

\section{Arterial lesion}

Although not very frequent, this can occur in proximal epiphyseal detachments of the tibia, injuring the anterior tibial artery. Arterial lesion provoked by spasm can be immediate or of late onset. 
It is extremely important for the physician assistant to watch out for the appearance of this severe lesion, as it can culminate in the amputation of the limb.

\section{Neurological lesion}

This can be observed in proximal and lateral lesions of the leg caused by injury of the fibular nerve in fibular neck fractures, in proximal tibiofibular dislocations and in exaggerated external compression in the production of the plaster cast. It is important for the physician assistant to search constantly for early signs of nerve compression with hallux extension and presence of paresthesias in the specific territories.

\section{LATE-ONSET COMPLICATIONS}

\section{Progressive Deformities}

Varus or Valgus Knee

These can occur in proximal epiphysometaphyseal fractures of the tibia by partial closing of the growth plate.

It should become a routine in clinical practice to warn the family about the possible appearance of this kind of complication whenever these fractures are present.

According to Koval et al. ${ }^{1}$ progressive valgus angulation can result from a combination of factors such as tearing of the perichondrial ring of the lateral shaft at the time of the lesion or the medial exuberant perichondrial callus formation, which results in excessive growth of the fractured bone. Other hypotheses include periosteum incarceration in the medial site of the fracture or concomitant lesion of the pes anserinus ("goosefoot"), which results in loss of the inhibitory effect of pinching on the physis, allowing excessive growth. The deformity is more prominent in the first year after the fracture and younger patients can present spontaneous correction with remodeling.

In relation to the treatment it was recommended, for example, to use resection or addition wedges in the plaster, with inversion of the removed fragment to correct the deformity.

In patients over the age of 12 years professionals have used dome osteotomy, which should be fixed with two crossed Steimann wires, plus plaster cast immobilization for 45-60 days.

Another technique in use is partial epiphysiodesis (lateral or medial), which depending on the residual deviation can be permanent by means of curettage or transitory with Blount staples or transfixing screws.

In distal metaphyseal fractures of the tibia, inadequate reduction or collapse due to caving of the fracture can result in a recurvatum deformity. Younger patients tend to tolerate this better, since remodeling in general makes the deformity insignificant. Older patients might need supramaleolar osteotomy when the recurvatum deformity is so severe it compromises ankle function and gait.

\section{HALT OF EPIPHYSEAL GROWTH}

The premature closure of the proximal tibial shaft ${ }^{1}$ can occur with unnoticed lesion by crushing (Salter-Harris V), resulting in growth suspension. It most usually affects the anterior shaft, which entails recurvatum deformity of the affected knee. The closing of the distal tibial shaft can also occur in undiagnosed "crushing" lesions (Salter Harris V) in the distal tibial physis, resulting in growth halt. Bone lengthening can be indicated in these cases, depending on the disparity in the residual length or on how much it will be predictable.

\section{WARNING}

Progressive varus and valgus deformities are likely to occur after proximal epiphysometaphyseal fractures. There can also be knee recurvatum due to the premature closure of the proximal tibial physis, resulting from an unnoticed lesion caused by crushing. Thus it should become a routine in clinical practice to warn the family of the possible appearance of post-traumatic deformities in the immature skeleton.

\section{ACKNOWLEDGMENT}

We are grateful to the Núcleo de Apoio à Publicação da Faculdade de Ciências Médicas da Santa Casa de São Paulo - NAP-SC for their technical and scientific support to the publication of this manuscript.

\section{REFERENCES}

1. Koval KJ, Zuckerman JD, Handbook of fractures. 3rd ed. New York: Lippincot Willians \& Wilkins; 2006.

2. Mann DC, Rajmaira S. Distribution of physeal and nonphyseal fractures in 2,650 long bone fractures in children aged 0-16 years. J Pediatr Orthop. 1990;10:713-6.

3. Rockwood CA Jr. Fractures in children. Philadephia: JB Lippincott; 1984.

4. Worlock P, Stower M. Fracture patterns in Nottingham children. J Pediatr Orthop. 1986;6:656-60.

5. Beaty JH, Kasser JR, editors. Rockwood and Wilkins fraturas em crianças. Tradução de Mirtes Frange de Oliveira Pinheiro. 5ª ed. São Paulo: Manole; 2004.

6. Weber BG, Brunner C, Freuner F. Treatment of fractures in children and adoles cents. Berlin: Springer-Verlag; 1980.

7. Rang M. Children`s fractures. 2nd ed. Philadelphia: JB. Lippincott Company; 1982.

8. Rockwood CA Jr. Fractures in children. $4^{\text {th }}$ ed. Philadelphia: Lippincott; 1996.

9. Zionts LE, MacEwen GD. Spontaneous improvement of post-traumatic tibia valga. J Bone Joint Surg Am. 1986;68:680-7.

10. Robert M, Khouri N, Carlioz H, Alain JL. Fractures of the proximal tibial metaphysis in children: review of a series of 25 cases. J Pediatr Orthop. 1987;7:444-9.

11. McCarthy JJ, Kim DH, Eilert RE. Posttraumatic genu valgum: operative versus nonoperative treatment. J Pediatr Orthop. 1998:18:518-21.

12. Tuten HR, Keeler KA, Gabos PG, Zionts LE, MacKenzie WG. Posttraumatic tibia valga in children. A long-term follow-up note. J Bone Joint Surg Am. 1999;81:799-810.

13. Shannak AO. Tibial fractures in children: follow-up study. J Pediatr Orthop. 1988;8:306-10

14. Hansen B, Greiff J, Bergmann F. Fractures of the tibia in children. Acta Orthop Scand. 1976:47:448-53.

15. Sarmiento A, Latta L, Zilioli A, Sinclair W. The role of soft tissue in the stabilization of tibial fractures. Clin Orthop Relat Res.1974;(105):116-29.

16. Yang JP, Letts MR. Isolated fractures of the tibia with intact fibula in children: a review of 95 patients. J Pediatr Orthop. 1997;17:347-51.
17. Muller E, Allgover Schneider R. Willenegger H. Manual of internal fixation. 3rd ed New York: Springer-Verlag; 1991

18. Srivastava AK, Mehlman CT, Wall EJ, Do TT. Elastic stable intramedullary nailing of tibial shaft fractures in children. J Pediatr Orthop. 2008;28:152-8.

19. Lascombes $P$, Haumont $T$, Journeau $P$. Use and abuse of flexible intramedullary nailing in children and adolescents. J Pediatr Orthop. 2006;26:827-34.

20. Vallamshetla P, de Silva U, Bache CE, Gibbons PJ. Flexible intramedullary nails for unstable fractures of the tibia in children. An eight-year experience. J Bone Joint Surg Br. 2006;88:536-40.

21. Kubiak EN, Egol KA, Scher D, Wasserman B, Feldman D, Koval KJ. Operative treatment of tibial fractures in children: are elastic stable intramedullary nails an improvement over external fixation? J Bone Joint Surg Am. 2005;87:1761-8.

22. El-adi G, Mostafa MF, Khalil Ma, Enan A. Titanium elastic nail fixation for paediatric femoral and tibial fractures. Acta Orthop Belg. 2009;75:512-2

23. Irwin A, Gibson $P$, Ashcroft $P$. Open fractures of the tibia in children. Injury. 1995:25:21-4

24. Kreder $\mathrm{HJ}$, Armstrong P. A review of open tibia fractures in children. J Pediatr Orthop. 1995; 15:482-8.

25. Cullen MC, Roy DR, Crawford AH, Assenmacher J, Levy MS, Wen D. Open fracture of the tibia in children. J. Bone Joint Surg Am. 1996;78:1039-47.

26. Song KM, Sangeorzan B, Benirrschke S, Browne RI. Open fractures of the tibia in children. J Pediatr Orthop. 1996;16:635-9.

27. Blazier RD, Barnes CL. Age as a prognostic factor in open tibial fractures in children. Clin Orthop Relat Res. 1996;(331):261-4.

28. Bartlett CS 3rd, Weiner LS, Yang EC. Treatment of type II and type III open tibia fractures in children. J Orthop Trauma. 1997;11:357-62.

29. Buckley SL, Smith GR, Sponseller PD, Thompson JD, Robertson WW Jr, Griffin PP. Severe (Type III) open fractures of the tibia in children. J Pediatr Orthop. 1996:16:627-34.

30. Gougoulias NE, Khanna A, Maffulin N. Open tibial fractures. Are children small adults. Hippokratia. 2009; 13:147-53 